\title{
Individual Mid-Air Gesture Sets Informed by Conceptual Metaphors: A Case Study on How Users Generate Mid-Air Gesture Sets to Control Video Streaming
}

\begin{abstract}
Recent research on mid-air gesture interaction for TV control aimed to standardize them. To this end, researchers developed a design approach that relies on the agreement rates among the elicited end-user gestures. Contrasting with the agreement based approach; a recent study have shown that the most common mid-air gestures might not be the most favored ones. In addition to this, researchers claimed that the agreement studies ignore users' cultural and conceptual bias. Thus, it can be postulated that the mid-air gesture interaction research can benefit from a qualitative analysis of the users' mid-gesture set design processes. Towards this end, this study investigated users' task conceptualizations and mental models. For this purpose, a mid-air gesture-based video streaming experiment was simulated with 10 participants, 4 females and 6 males. Through the lens of Conceptual Metaphor Theory, the study investigated the similarities between the participants' conceptual representations. The study findings demonstrated that the participants' conceptualizations had clear references to their bodies and prior physical experiences with the objects, which reflected as linguistic representations of orientational and ontological metaphors in participants' explanations. Further findings of the study addressed intersections between participants' mental models.
\end{abstract}

\section{Keywords}

Mid-air gesture interaction, conceptual metaphors, The Wizard of Oz method, video streaming experience

\section{Introduction}

TV and streaming technologies have advanced significantly in the last 10 years. With the rise of interconnectivity, streaming platforms have revolutionized the TV experience. The number of streaming platform use per household has surpassed that of cable TV use in some countries [1]. The evolution in content accessibility has reinforced the idea of redesigning our interaction with TV [2]. Research on novel TV control systems projects a future interaction experience that surpasses the limits of graphical interfaces. Research in the Human-Computer Interaction field has highlighted that mid-air gesture interaction may deliver the desired "natural" interaction experience. However, researchers have found that the standardization of the mid-air gesture interaction was problematic. In pursuit of a standardized mid-air gesture vocabulary, several elicitation studies were conducted. These studies aimed to find the most recurring mid-air gestures to design the gesture sets. In this way, the produced mid-air gestures could become the archetypes of their category. However, in a recent 
study, researchers postulated that the agreementbased approach ignores the users' conceptual, cultural and physical bias [3]. Thus, presented agreement calculations were inflated. Moreover, another group of researchers found that the mid-air gesture sets can vary across cultures and that the most common gestures might not provide the best design solutions [4]. Thus, it can be speculated that the most suitable design approach for mid-air gesture set has not yet been formulated. The underlying reason for this problem may be the prevailing gesture consensus approach in such studies because the approach neglected the importance of visual references and assumed that the human interpretation of the outputs and verbal commands could be standardized. Moreover, the method also postulated that the users could always design coherent, engaging mid-air gesture sets in a limited time period. Alternatively, in a recent research, a turn towards a human-centered design approach has been suggested [4]. The study evaluated users' appreciation for all of the mid-air gestures regardless of the agreement rates and found that the most agreed gestures may not be the most appreciated ones. In line with this imperative debate on the design approach, this study aimed to get a better understanding of the users' mid-air gesture set design processes. With this aim, a computeroperated streaming control experience was conducted with 10 participants. Throughout the experiment, the participants controlled a streaming activity with their mid-air gestures, and after demonstrating each gesture, the participants explained their thought processes. Later in the study, with the guidance of Conceptual Metaphor Theory (CMT), the users' conceptual representations for the given tasks, semantic similarities between these conceptual representations, the users' task grouping behaviors, and intersecting mental models were investigated. By doing so, the study evaluated the possibility of employing a mental-model-based design approach. The contributions of this study to the design field are (1) presentation of the collected mid-air gesture sets for streaming control, (2) examination of the conceptual representations of the tasks, (3) presentation of shared conceptual representations of the tasks and participants' intersecting mental models and (4) suggestions for the design of mid-air-gesturebased streaming experience.

\section{Background Work}

\subsection{Conceptual Metaphor Theory (CMT)}

Metaphors are commonly used to explain one thing in terms of another. In 1980, Lakoff and Johnson proposed through their CMT that humans' conceptual thought processes are comprised of metaphors [5]. Metaphors reflect the diversity of our cultural, physical and cognitive experiences. Based on how they are formed, Lakoff and Johnson categorized conceptual metaphors as structural, ontological and orientational.

A structural metaphor enables us to define one concept in terms of another concept. For instance, "Argument is War" metaphor uses "Argument" and "War" as concepts. To understand which aspect of the "War" is assigned to the "Argument" concept, we employ the most defined qualities of the "War" concept. Ontological metaphors differ from the structural metaphors in the nature of their source domains. The source domains of ontological metaphors are concrete objects, tangibles, and entities. For instance, "The mind is a machine" metaphor uses the "machine" as its source domain and assigns its qualities to the "mind" concept. In this way, the mind can be explained with the qualities attributed to a machine.

Structural and ontological metaphors show similarities in the way they are formed. Both of them explain unfamiliar concepts in terms of other familiar domains. However, in some cases, we explain the concepts in terms of relationships. Orientational metaphors are formed by relying on these relationships. They define a whole system of concepts in terms of spatial relationships, such as up-down, deep-shallow, or on-off. For instance, "having control over a situation" is based on the conception that "force is up".

In summary, CMT advocates that direct, embodied physical experiences can shape our conceptual thinking [6]. As mid-air gesture interaction relies on bodily communication, herein it is postulated that CMT can guide us to decipher the source of conceptualizations represented by mid-air gestures. Although Conceptual Metaphor Theory stands on linguistic evidence, research on cognitive science demonstrated that both linguistic and bodily expressions are resourceful in explaining our conceptualization [7]. Hence, in this study, the 
analysis of users' self-reported statements and gestural expressions were employed to unfold the conceptual representations in the participants' mid-air gestures. The analysis of the collected materials benefited from CMT in classifying the participants' recurring conceptual representations and gesture grouping behaviors.

Classified conceptual representations depicted the type of sources that users consulted while forming their midair gestures.

\subsection{Gesture Taxonomy and Agreement Based Approach}

Previous research on mid-air gesture interaction for TV control systems has focused on eliciting end-users' gestures and proposing methods to design intuitive gesture sets. In the last 5 years, $74.5 \%$ of studies have employed Wobbrock et al.'s "guessability method" to design the most intuitive gesture sets [8]. The remaining studies have used "choice-based elicitation", "Nielsen's intuitive and ergonomic model", "a combination of Wobbrock's and Nielsen's models" and other methods [8]. Due to the prevalence of Wobbrock et al.'s classification model and guessability method, this section explains them in detail.

According to Wobbrock et al., gestures can be classified based on four dimensions: their form, nature, flow, and their binding elements. These dimensions are used in defining a gesture's movement such as static, dynamic and one point; a gesture's relationship with the object and space around it, such as object dependent, world dependent, world independent and mixed dependencies; the continuity of the gesture to be recognized, such as continuous or discrete; and the semantics of the gestures, such as symbolic, physical, metaphoric and abstract [9]. Symbolic gestures refer to the symbolic representations, for e.g., the well-known "OK" and "STOP" poses. Physical gestures are demonstrations of the actual physical interaction with the objects on screen/in the air [9]. Metaphorical gestures rely on semantic associations between the gestures and delivered functions. Abstract gestures are the remaining gestures that do not convey any association with the referred function [8]. Often, based on this taxonomy, researchers employed end-user elicitation studies and guessability method to standardize mid-air gesture sets. In a typical elicitation study, participants are directed to produce mid-air gestures for certain referents and depending on the employed study method, the most recurring or correctly guessed mid-air gestures become the representative of the commands (referent). However, recent research has demonstrated that agreement rates across individuals' mid-air gestures could be quite low in studied samples; moreover, mid-air gesture sets could vary across cultures [4]. In addition to that, the most common mid-air gestures might not indicate that it would be the most appreciated one [4]. Therefore, it is not clear how mid-air gesture sets should be designed. In this sense, users' conceptualization processes can help formulate a better design approach. Towards this end, the present study aimed to understand the type of conceptual representations users deployed to form their mid-air gestures.

\subsection{Mid-Air Gesture Interaction for Interactive TV Control}

First introduced by Freiman and Weisman, dynamic hand gestures are now being proposed as a novel way of interaction with TV [10]. Since the emergence of dynamic hand gestures, several studies investigated the possibility of redesigning interactive TV experience with mid-air gesture interaction. For instance, studies by Zaiti et al. and Wu et al. investigated users' mid-air gesture preferences for TV functions, and Vavatu et al. compared user agreement rates between hands-free interaction and augmented remote control interaction [2], [11], [12]. Further research on mid-air gesturebased TV control examined the possibilities of handsfree text entry for Interactive TVs and preferences for mid-air gesture control among older adults' [13], [14]. Research on mid-air gesture interaction for TV frequently progressed through end-user gesture elicitation and choice-based elicitation studies. Research in this field constantly aimed to standardize mid-air gesture-based TV interaction often relying on the consensus of the collected mid-air gestures.

\section{Experiment}

\subsection{Participants}

Ten participants (F: 4, M: 6, Median: 31), of ages ranging from 29 to 33 years, volunteered for the present study. Their professions varied: three aerospace engineers, 
Fig. 1. An Illustration of the Experiment Setup

Fig. 2. Gestures of the study participants for the "Turn On" Command and the metaphorical references in gestures

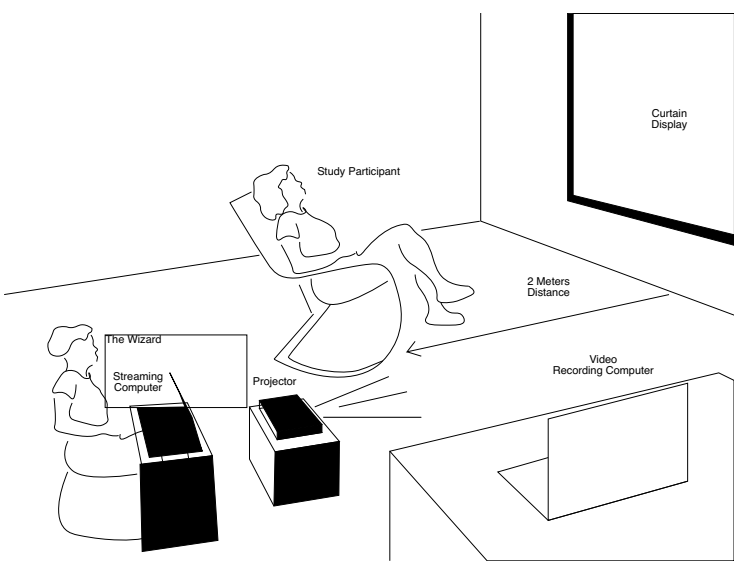

three mechanical engineers, one system engineer, one mathematics teacher, and two psychologists. The main criteria for the selection of this participant group were that (i) the participants reported prior on-screen gesture experience ranging between six to eight years;

(ii) frequent use of online video streaming services; (iii) they had no prior experience with mid-air gesture control systems; and (iv) they were motivated to perform the necessary tasks.

\subsection{Procedure}

Prior to the study, the participants were briefed about the mid-air gesture interaction. The study took place in a home environment, which the participants visited 1 to 3 times per week. The participants' familiarity with the household could assure that the experiment was close to a real experience. On their arrival, the participants were informed once more that they could use either a single hand or both hands to perform the mid-air gestures. The test apparatuses were a digital projector, a projection screen $(2.6 \times 2$ meters $)$, a speaker and a laptop, the use of which the participants were already familiar with. The study was performed using the Wizard of Oz method, wherein the participants were instructed to interact with hypothetically autonomous subjects [15]. Throughout the experiment, the wizard sat behind the participants so that she would not influence the participants' gesture design process, as shown in Fig.1. Participants performed the announced tasks using mid-air gestures, and after the announcements of the tasks, the wizard simulated the effects. After each gesture, the participants explained what inspired their gestures. The wizard operated the "YouTube" video streaming platform in full-screen mode in this case. The choice of the video platform was based on the participants' familiarity with it. In this context, the participants generated mid-air gestures for the announced tasks in the following order: "turn on the laptop", "turn on the speaker", "connect/pair the devices”, "play”, “pause”, "adjust the volume”, "adjust the speed", "adjust the video quality", "subtitles on", "subtitles off" and "watch later". With the consent of the participants, the wizard recorded the whole process using a MacBook Pro in-built camera.

\section{Study Findings}

\subsection{Conceptual Representations of the Commands}

This section presents the analysis of the conceptual representations of the commands (referents) reflected in the participants' mid-air gestures.

“Turn On". Firstly, the participants performed mid-air gestures to give the "Turn on" command to the laptop computer. To "Turn on" the laptop, Participant 2 (P2) demonstrated a "greeting" gesture. P4 and P8 used the transition of their palms from a closed state to open state, and the gesture was supported by the rise of their arm along a diagonal axis. P4 and P8 reported that they used "visible, up and ready palm" to represent "being on". P6, P7, and P10 also demonstrated the "rise

\section{GESTURES FOR “TURN ON” COMMAND - COMPUTER}

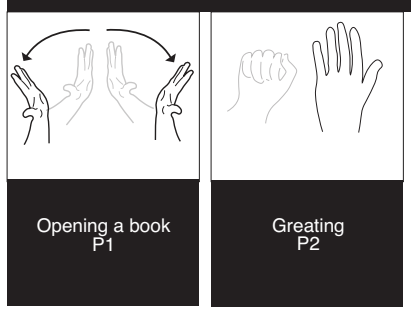

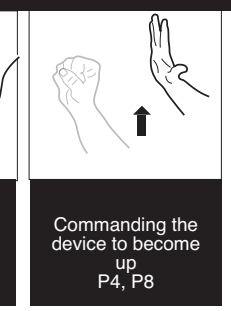
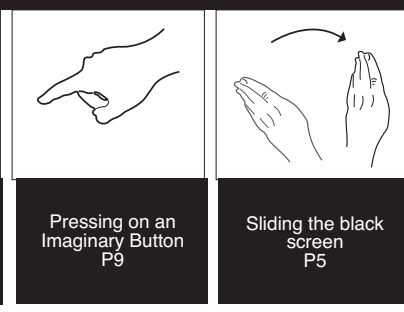

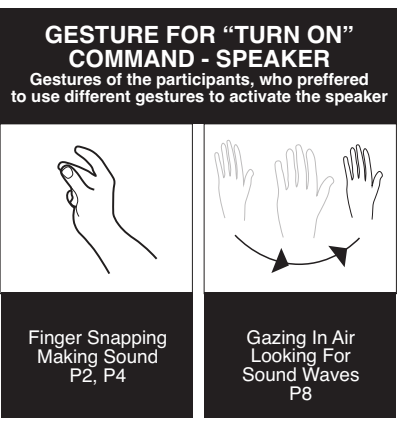




\section{GESTURES FOR THE "PAIR" COMMAND}
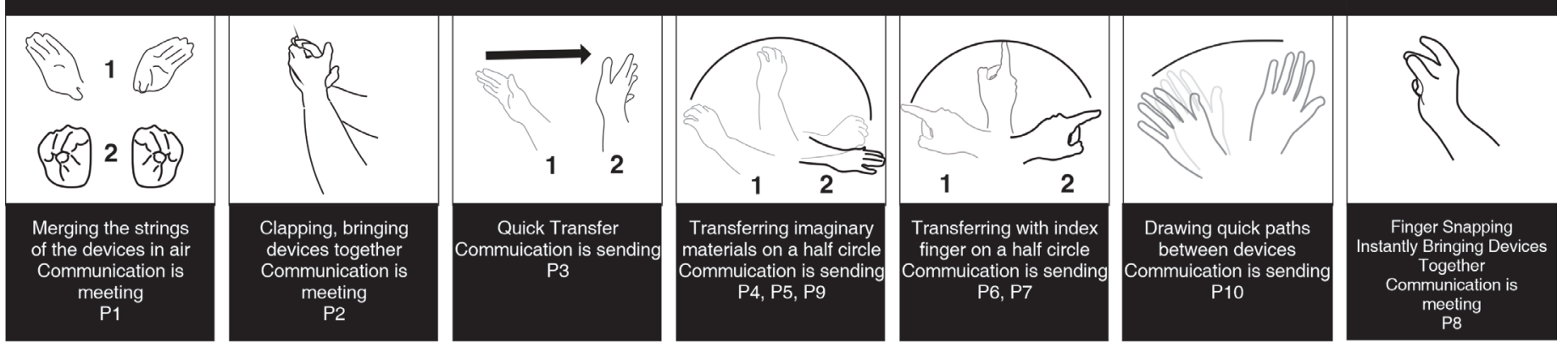

\& stand up" movements in air with their palms facing upwards, rising along the vertical axis. Conceptual representations of the tasks in 6 participants' mid-air gestures correlated with the orientational metaphors defined by Lakoff as "conscious is up" and "force is up" [5]. Of the remaining four participants, P3 and P9 delivered the command by interacting with imaginary buttons. P1 demonstrated two open facing palms extending along the horizontal axis and reported that the movement depicted "opening a book". P5 swiped her right palm starting from her left to the right along the horizontal axis. The gestures are summarized in fig.2. Later, the participants were also asked to turn on the Bluetooth speaker with mid-air gestures. P2, P4, and P8 mentioned that the speaker would require different mid-air gestures since its predominant purpose was playing music, while the remaining participants maintained the same mid-air gestures for both devices. P2 and P4 snapped their thumb and middle fingers, given that since ancient times, snapping fingers had represented tracing the rhythm [16]. Thus, it can be speculated that the participants referred to the speaker as a company, "who" is capable of responding to them. P8 depicted an open palm slowly moving along the peripheral axis from left to right in front of her upper torso and explained that the movement represented "gathering sound waves".

"Pair". As the third task, the participants were asked to use mid-air gestures to "pair" the laptop and Bluetooth speaker. The participants were informed that the devices' Bluetooth function was on in its default mode. As shown in Fig.3, to connect/pair the devices, P6 \& P7 traced a half circle between the objects using their index fingers and P4 \& P9 traced a half circle between the objects with their palms. P3 and P5 swiped a single open palm along the horizontal axis and P10 used a single palm facing downwards to wave between two imaginary points. These participants' self-reported explanations indicated that the gestures represented a hypothetical entity is sent through a half-circle or a horizontal path between the devices. Conceptual representations of the task correlated with "communication is sending" metaphor. The remaining participants executed clapping, finger snapping and gradually approaching palm gestures. These participants mentioned that the movement signified "the union of the devices".

"Play". To play the video, of the ten, six participants directly pointed to a random spot on the screen, and of them, only P1 avoided using the index finger on its own for the pointing gesture. Instead, he used his index and middle fingers firmly held together and pointed at the play button as if he was firing a gun. P7 used a fingersnapping gesture, P5 and P8 used the rising opening palm gesture, P2 moved his finger up and P10 swiped his hand to the right. In general, the participants used pointing gestures and performed the gesture in a quick discrete movement. However, P2, P5 and P8's gestures correlated with Lakoff's "active is up" metaphor.

"Pause". Of the ten participants, five used the pointing gesture to give the "Pause" command. One participant closed her palm to a shrunk position and one participant used the finger snapping gesture. Two participants used the static open palm pose and one participant moved down his finger. In general, the participants either repeated the same gestures they used to give the "Play" command or demonstrated the reverse motion. Additionally, two participants used the static "Pause" pose.
Fig. 3. Gestures of the study participants for the "Pair" Command and the metaphorical references in gestures 


\section{GESTURES FOR "PLAY" COMMAND}

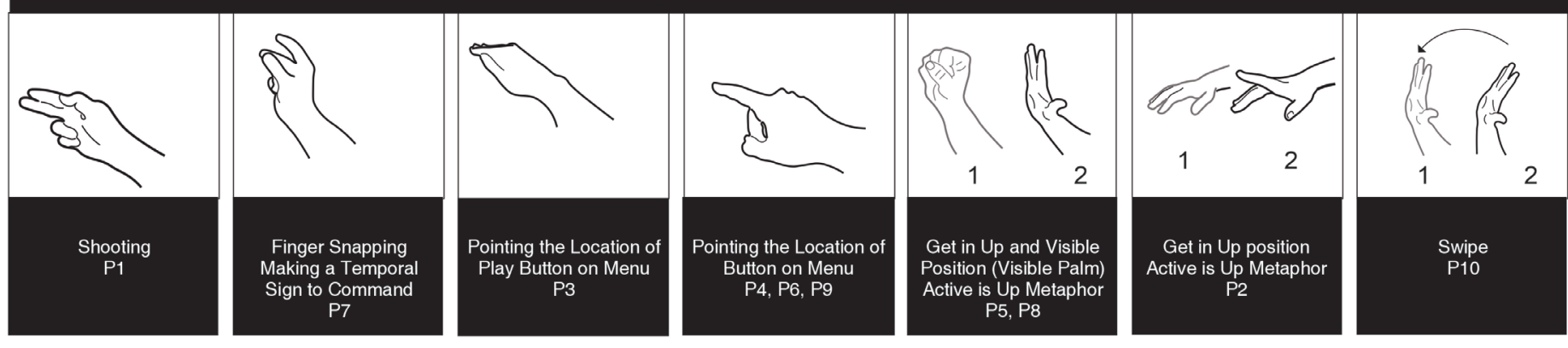

\section{GESTURES FOR "PAUSE” COMMAND}

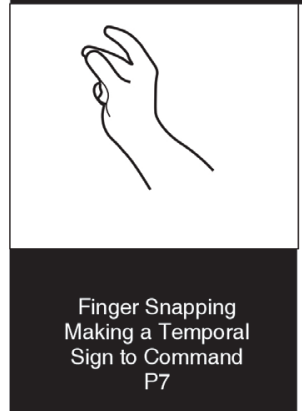

Fig. 4. Gestures of the study participants for the "Play" \& "Pause" commands and the metaphorical references in gestures
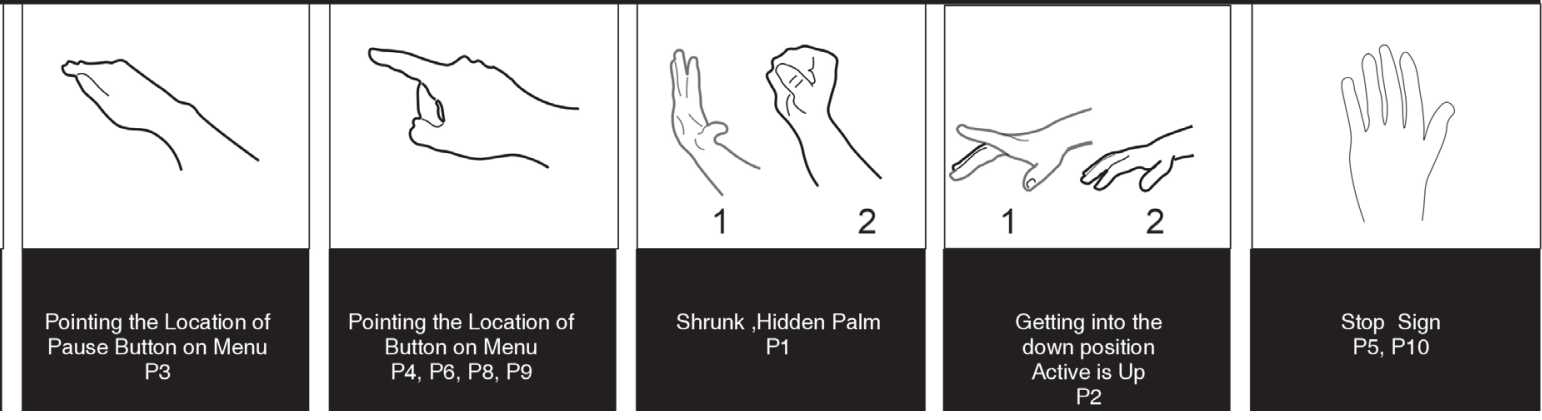

Volume Adjustment Control. All of the participants demonstrated their gestures along the vertical axis. They either moved an index finger or a single palm along this axis. Only P1 moved his finger around his ear to adjust the volume; however, even he used the vertical axis for adjustment. The participants reasoned that spatial highness represents more. Previously, in embodied music cognition, it is reasoned that vertical representation of the sound amplitude aligns with "greater is higher" metaphor [17]. The findings of this study correlated with this claim, as all of the participants used the vertical axis for volume adjustment.

Speed Adjustment Control. The experiment continued with speed adjustment command. P3 relied on his prior on-screen gesture experience, and consequently, used the double-tap gesture. P9 directly pointed his fingers to the screen. P7 used finger snapping, P2 tapped on his face and P4 used the swiping gesture. P5 tapped and bounced her hand periodically on the horizontal axis while P1, P6, $\mathrm{P} 8$, and P10 illustrated a periodic cyclic motion. All except $\mathrm{P} 3$ and $\mathrm{P} 9$ adjusted the speed of their hand motions to demonstrate the speed required. Except for the pointing gestures, the participants' mid-air gestures were diverse, yet coherent. All of the gestures demonstrated a "periodic" motion either along a linear or cyclic path. The participants associated the command with the motions of "bouncing", "swirling", "swiping" and "rolling". Some of the participants who demonstrated a cyclic motion reported that cassette tapes inspired their mid-air gestures. To give the reverse commands, the participants just reversed the directions.

Video Quality Adjustment. Five participants pointed to the screen to adjust the video quality. P6 moved her right open palm along the vertical axis as she previously used her left hand for the sound adjustment. The remaining participants demonstrated varying mid-air gestures. P2 moved his thumb in "OK" position along the vertical axis. P5 demonstrated a gesture that resembled drawing a " $V$ " shape in the air and she reported that the gesture is inspired by the "sunrise" metaphor. P7 tapped on his eye to adjust the video quality and $\mathrm{P} 8$ rubbed her palms on a hypothetical surface in air. P7 reported that he used pointing the eye as an indicator of better vision and P8 reported that rubbing surface refers to clearer vision. These midair gestures were semantically associated with vision. Vision is often associated with brightness and clearance, 

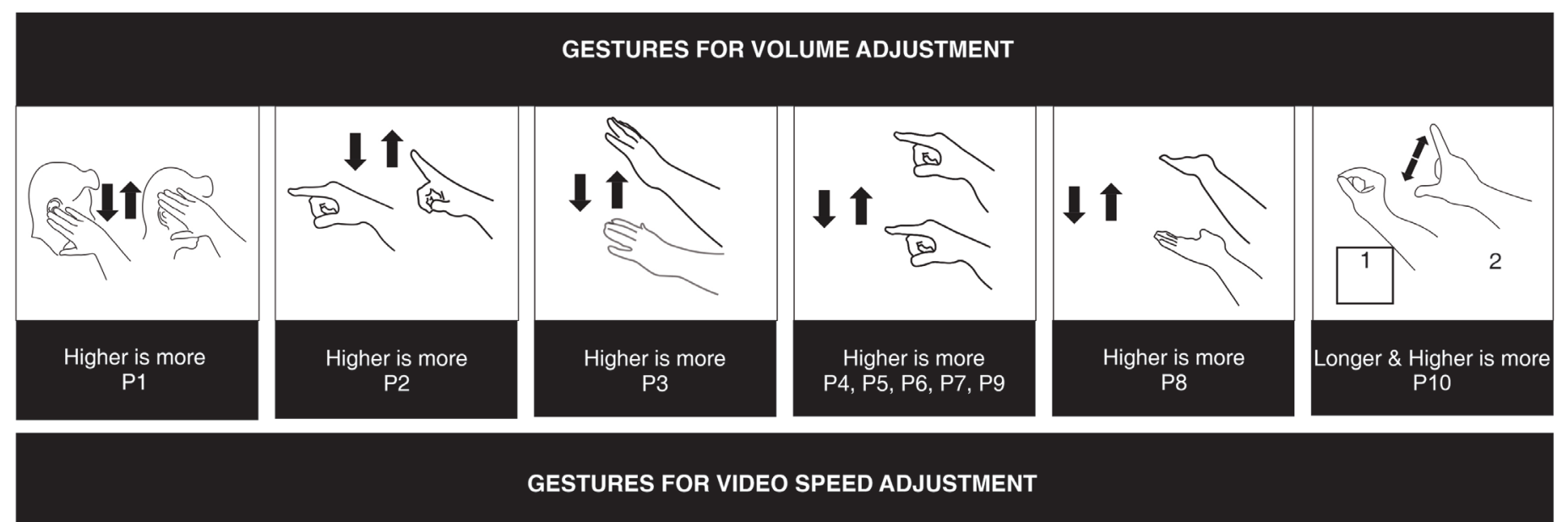

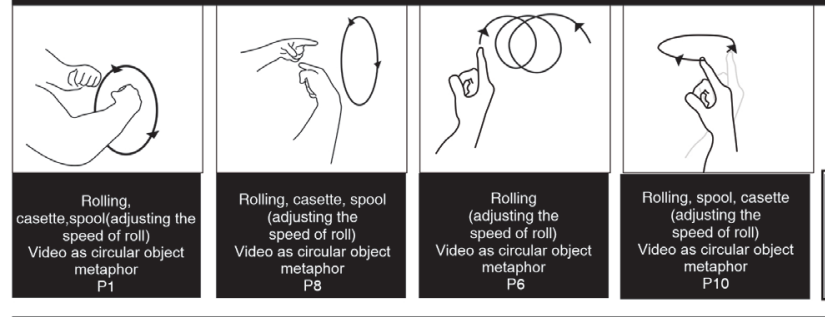

GESTURES FOR VIDEO QUALITY ADJUSTMENT
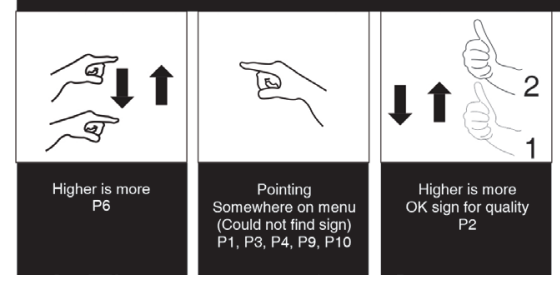

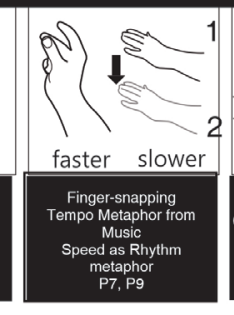

P9
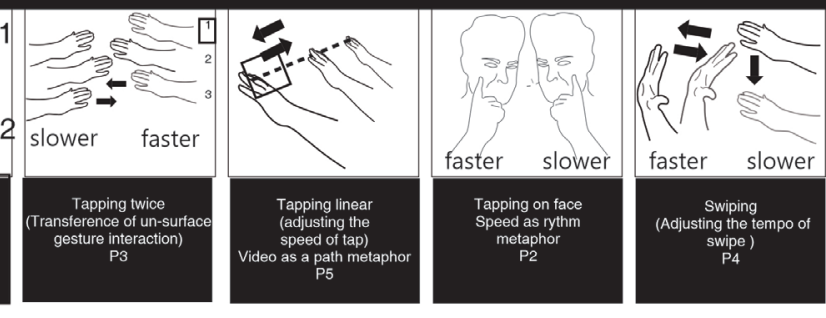

GESTURES FOR "WATCH LATER" COMMAND
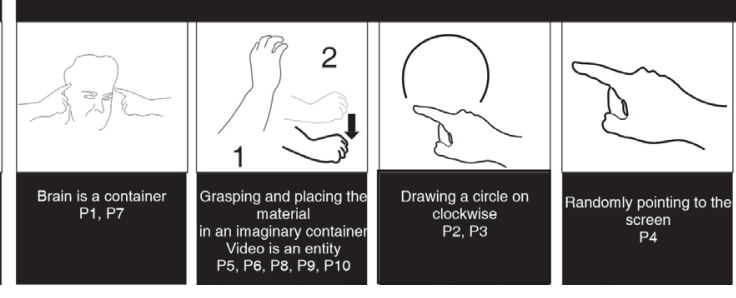

and the references were coherent with the commands [18]. Interestingly, the study participants had difficulty in finding gestures to adjust the video quality. All of them asked for extra time to find a mid-air gesture.

"Watch Later". To provide the "watch later" command, two participants directly pointed to the graphical interface and swirled their index fingers in the clockwise direction. Two participants pointed to their skulls with their index fingers. These participants reasoned that pointing to the skull meant storing the information in the brain. Four participants grasped the content with a single hand and placed it in an imaginary container. One participant reported that she perceived the content as a shopping item. Conceptual representations of the command indicated that the content is often perceived as a physical entity that can be grasped and stored in a container.
“Turn On \& Off the Subtitles". To turn on the subtitles, P1 and P4 used pointing gestures. P3, P7, P8, $\mathrm{P} 9$, and $\mathrm{P} 10$ swiped their right hand or fingers along the horizontal axis. The position of their hands emphasized the location where the subtitles were often placed on video players. Three participants demonstrated gestures that did not have any reference to the graphical interface. $\mathrm{P} 2$ revolved his right palm from downward to upward direction and rationalized that "facing up" means "being on". P6 used typing gesture with both hands moving along the vertical axis to turn on \& off the subtitles. P5 grasped an imaginary entity with her hand. Subtitle control gestures were often physical. However, the representation of "active is up" metaphor was visible in $\mathrm{P} 2$ and $\mathrm{P} 6$ 's gestures. Additionally, $\mathrm{P} 5$ 's gesture represented "understanding is grasping" metaphor.
Fig. 5. Gestures of the study participants for the "Volume Adjustment" "Sound Adjustment", "Quality Adjustment" and "Watch Later" commands and the metaphorical references in gestures 


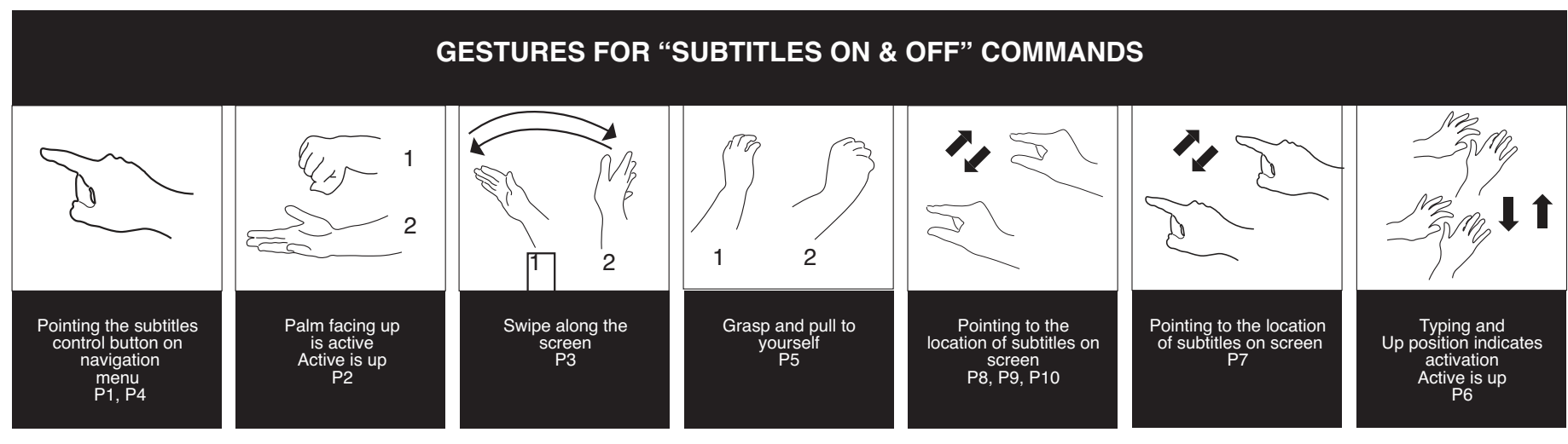

Fig. 6. Gestures of the study participants for the "Subtitles

On" \& "Subtitles Off" commands and the metaphorical references in gestures

\subsection{Shared Conceptual Representations of the} Commands

The participants' shared conceptualizations of the tasks are summarized as follows:

Turn on the Laptop. "Force is up" metaphor. Turn on the Speaker. The personification of the speaker. The participants referred to the speaker as "a person/entity that can respond to sound".

Pair the Devices. "Communication is sending" and "communication is meeting" metaphors.

Play \& Pause. "Active is up" metaphor.

Adjust Volume. "Higher is greater" metaphor.

Adjust Video Speed. Representation of the video as "a rolling circular entity" and "speed as rhythm".

Adjust Video Quality. The shared conceptualization correlated with "higher is greater" metaphor, however, the participants also employed interesting metaphors such as "video as a surface", "visual quality as sunrise" and "the eye as a button". P6 used "Greater is higher" metaphor to link the task with the volume adjustment task.

Turn on the Subtitles. The participants agreed on pointing towards the typical location of subtitles on graphical interfaces.

Watch Later. "Video as a graspable entity" and "brain as a container" metaphors.

\subsection{Intersecting Mental Models}

Pointing Gestures for Relatively Complex

Commands. According to the study results, the participants used pointing gestures when they had difficulty in finding suitable physical demonstrations. A study by Mauney et al. found similar results [19]. However, the "Play" command can be an exception to this generalization since the participants used pointing gesture in a distinguishable way.

\section{Reverse Gestures for the Dichotomous}

Referents. Previous studies have shown that the users often preferred reversible gestures for the dichotomous referents and the findings of this study supported this inference [2], [9]. This behavior often surfaced in the demonstrations of physical experiences.

Same Gestures for the Dichotomous Referents. Study findings have shown that some of the participants employed the use of same gestures for dichotomous referents, such as for "Play / Pause" and "Subtitles on/ off" commands. The participants who employed this model associated these commands with clicking the buttons. Our findings support the findings of a previous study by Kühnel, C. et al. [20].

\section{Redefining the Same Gesture with the "Stop"} Pose for Dichotomous Referents. Even though the participants were encouraged to use a single gesture, three participants used the static "stop" pose and "OK" pose at the end of certain gestures.

Limited Hand/Finger Movements. Two participants tried to limit their hand/finger use. P9 used her right index finger for most of the commands and P2 placed his fingers on the armrest, which restricted his motions.

\section{Transferring On-Screen Gestures to Mid-Air}

Gesture Interaction. Our findings have shown that a few participants used on-screen gestures as their midair gestures. 


\section{Discussion}

This study aimed to understand how users generated their mid-air gestures through the analysis of their conceptual representations. The motive of the study was to take a step back from agreement calculations and understand the recurring patterns in users' mid-air gesture set design processes. Study findings showed on a conceptual level there were explicit similarities among the participants' mid-air gestures. Study findings indicated that the participants frequently represented orientational and ontological metaphors with their mid-air gestures. As aforementioned, orientational metaphors rely on spatial relations between the concepts. Ontological metaphors enable us to explain concepts in terms of tangibles. When controlling the streaming activity, the participants often described their conceptual representations as ontological and orientational metaphors. Thus, it can be speculated that the physical experiences with the objects and the embodied experiences played a central role in the design processes. However, when the commands did not referphysical experiences, e.g. video quality adjustment, the participants' conceptual representations often relied on structural metaphors. Based on these findings, some of the suggestions to the designers can be summarized as follows:

\section{Participants' Physical Experiences with Objects} Inspires Mid-Air Gestures. The participants enjoyed depicting imaginary versions of physical experiences, such as commanding the computer to stand up, rolling the content as if they were rolling a physical wheel, grasping the content as if they were grasping an object and elevating the volume as if they were piling up things. Sometimes, they directly referred to their bodies. Thus, further explorations in mid-air gesture interaction should address physical interactions with different objects and how we refer to our bodies.

\section{Gesture Sets should be Recognizable when Performed in Different Volumes. Some of the} participants purposefully used minimal mid-air gestures. However, older adults may not have the same physical abilities as young people do to perform exaggerated mid-air gestures. Thus, mid-air gesture sets should provide a ranging volume use.

\section{On-screen Gestures may not Produce the} Desired Engagement. Despite that the volume adjustment uses a horizontal bar on the mobile graphical interface, participants preferred vertical movement for volume adjustment. Thus, a simple transfer of on-screen gestures to in-air ones may not produce equal engagement in users.

\section{Conceptualizations are Grouped in Terms of Spatial, Temporal and Semantic Aspects.}

To evaluate the wholeness of the designed mid-air gesture sets, gestalt principles can be resourceful. The participants employed spatial, temporal and semantic references to group their mid-air gestures. For instance, some participants relied on "greater is higher" metaphor to group "adjust volume" and "adjust video quality" tasks. Both tasks had different semantic references, however, were grouped in terms of their adjustability along the vertical axis. In such cases, the proximity of the semantic relationships between the conceptual representations should be studied further. The proximity built instantaneously by the participants may be misleading in the design process.

\section{Enable Customization. The designed gesture} sets should enable users to add mid-air gestures to the provided sets. In this way, the system becomes adaptable and users can feel in control of their gesture sets.

\section{References}

1. Richter, F. (2019). Infographic: Netflix Surpasses Major Cable Providers in the U.S. Statista Infographics. Retrieved 31, January, 2019 from https://www.statista.com/chart/9799/ netflix-vs-cable-pay-tvsubscribers/

2. Zaiți, I., Pentiuc, S., \& Vatavu, R. (2015) "On Free-hand TV control: Experimental Results on User-elicited Gestures with Leap Motion". Personal and Ubiquitous Computing, 19(5-6), 821-838.

3. Tsandilas, Theophanis. (2018) "Fallacies of Agreement: A Critical Review of Consensus Assessment Methods for Gesture Elicitation." ACM Trans. Comput.-Hum. Interact. 25(3)

4. Wu, Huiyue, et al. (2018)"The Gesture Disagreement Problem in Free-hand Gesture Interaction." International 
Journal of Human-Computer Interaction, 1-13.

5. Lakoff, G., \& Johnson, M. (2008) Metaphors we live by. University of Chicago Press.

6. Ritchie, L. D. (2006). Context and connection in metaphor. Springer, 31-57

7. Gu, Yan, et al. (2017). "Conceptual and lexical effects on gestures: the case of vertical spatial metaphors for time in Chinese." Language, Cognition and Neuroscience 32.81048 1063.

8. Vogiatzidakis, P., \& Koutsabasis, P. (2018) "Gesture Elicitation Studies for Mid-Air Interaction: A Review." Multimodal Technologies and Interaction 2(4)

9. Wobbrock, J.O., Meredith Ringel Morris, and Andrew D. Wilson. (2009) "User-defined gestures for surface computing." Proceedings of the SIGCHI Conference on Human Factors in Computing Systems. ACM.

10. Freeman, W. T., \& Weissman, C. D. (1995). "Television Control by Hand Gestures". IEEE International Workshop on Automatic Face and Gesture Recognition.

11. Wu, H. \& Wang, J. (2012) "User-defined body gestures for TV-based applications." 2012 Fourth International Conference on Digital Home. IEEE.

12. Vatavu, R. (2013) "A comparative study of user-defined handheld vs. freehand gestures for home entertainment environments." Journal of Ambient Intelligence and Smart Environments 5(2), 187-211.

13. Ren, G., \& O'Neill, E. (2013) "Freehand gestural text entry for interactive TV." Proceedings of the 11th European conference on Interactive TV and video. ACM.

14. Bobeth, J. et al. (2012) "Evaluating performance and acceptance of older adults using freehand gestures for TV menu control." Proceedings of the 10th European Conference on Interactive TV and Video. ACM

15. Bella, M. \& Hanington, B., (2012) Universal Methods of Design, Beverly, MA: Rockport Publishers.

16. West, M.L. (1992) Ancient Greek Music, Oxford University Press.

17. Cox, A. (2016) Music and embodied cognition: Listening, moving, feeling, and thinking Indiana University Press.

18. Coulson, S. \& Lai, V. (2016) "The Metaphorical Brain." Frontiers in human neuroscience 9, 699.

19. Mauney, D. et al. (2010) "Cultural similarities and differences in user-defined gestures for touchscreen user interfaces." CHI'10 Extended Abstracts on Human Factors in Computing Systems. ACM.

20. Kühnel, C. et al. (2011)."I'm home: Defining and Evaluating a Gesture Set for Smart-home Control" International Journal of Human-Computer Studies 69(11), 693-704. 